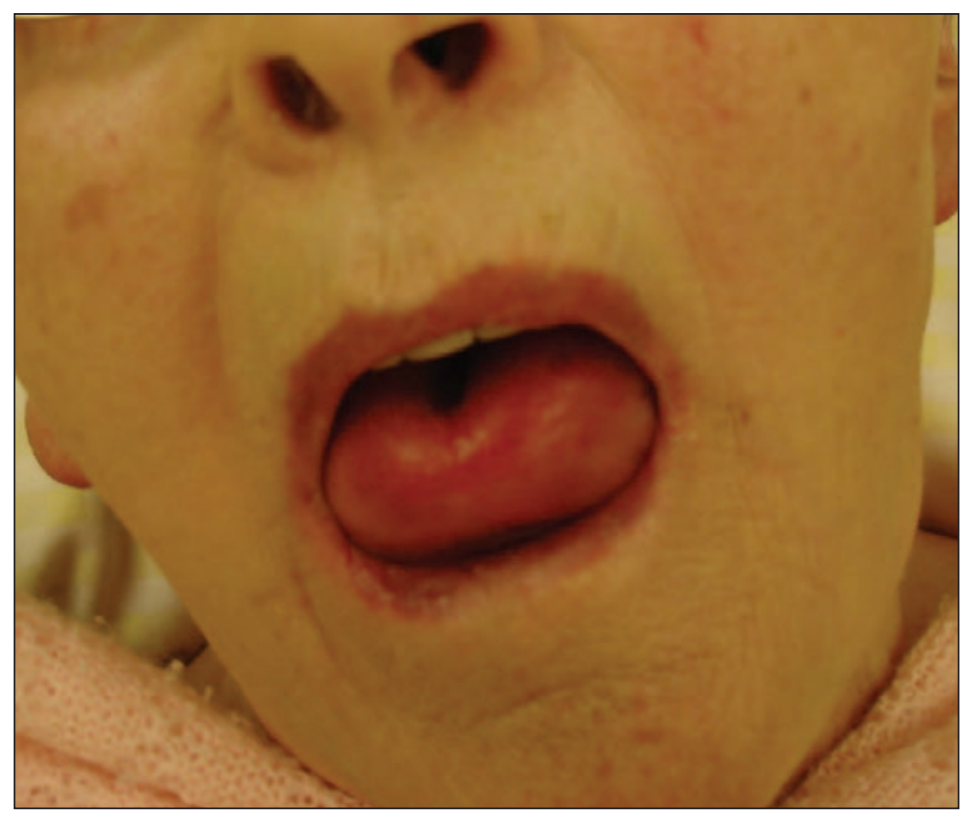

Figure 1: An 86-year-old woman with acute, painless, nonpruritic tongue swelling.

\title{
Angioedema of the tongue
}

A n 86-year-old woman presented with acute, painless, nonpruritic tongue swelling (Figure I) that had developed 6 hours earlier. She had no symptoms of upper airway obstruction or dyspnea, but her speech was dysarthric. The results of a physical examination were normal, and she had no urticaria. The patient was hypertensive, and she had been taking an angiotensin-converting enzyme (ACE) inhibitor (benazepril) for 12 years. We diagnosed ACE-inhibitor-induced angioedema and discontinued benazepril therapy. She was given intravenous steroids and antihistamines. There was no initial improvement, but within 2 days her tongue returned to normal size. The patient was given a different antihypertensive medication, and she remained symptom-free several months later.

Angioedema is acute, self-limited localized swelling of subcutaneous or mucosal tissue. It often affects the lips, eyelids, face, tongue, larynx or bowel, and often causes large, welldemarcated lesions that typically resolve in 2-3 days but may last 5-7 days. Angioedema affects $0.1 \%-0.5 \%$ of patients taking ACE inhibitors and may develop hours or years after therapy is initiated. Its onset may be idiopathic or the result of diverse causes such as nonsteroidal anti-inflammatory drugs, antibiotics, insect bites, food or environmental allergens and complement-inhibitor deficiencies. Cross reactivity with other types of ACE inhibitors is common, and a change of drug classes is necessary to prevent a recurrence. Treatments for angioedema are dependent on the underlying cause and may include antihistamines, corticosteroids, epinephrine and tranexamic acid.

CMAJ invites contributions to Interesting images, a new column with a very brief but clear description of the case, the images and the main teaching point. Submit manuscripts online at http://mc.manuscriptcentral.com/cmaj.
Florim Cuculi MD

Yves Suter MD

Paul Erne MD

Department of Cardiology Kantonsspital Luzern Switzerland 